\title{
Characteristic Brain Imaging Findings in a Patient with Joubert Syndrome and Segmental Glomerulosclerosis
}

\author{
Ahmed H. Abduljabbar, MBBS, SB-RAD \\ Department of Diagnostic Imaging, Faculty of Medicine \\ King Abdulaziz University, Jeddah, Saudi Arabia
}

\section{Correspondence}

Dr. Ahmed H. Abduljabbar

P.O. Box 80215, Jeddah 21589, Saudi Arabia

e.M: aabduljabar@kau.edu.sa

Submission: 21 Dec. 2014

Accepted: $\quad 22$ Feb. 2015

\section{Citation}

Abduljabbar AH. Characteristic brain imaging findings in a patient with joubert syndrome and segmental glomerulosclerosis. JKAU Med Sci 2015; 22 (2): 53-57. DOI: 10.4197/Med. 22.2.8

\begin{abstract}
Joubert syndrome is a rare disorder inherited as an autosomal recessive trait. It has distinctive clinical features and specific brain imaging findings on magnetic resonance imaging. Patients present clinically with hyperpnea, apnea, ocular abnormalities, developmental delay and truncal ataxia. In this case report, we illustrate a patient with Joubert syndrome and chronic renal failure. Magmetic resonance imaging examination revealed the characteristic "molar tooth" appearance of the brain stem, "bat-wing" shaped 4th ventricle, cleft vermis and deep interpeduncular fossa consistent with the diagnosis.
\end{abstract}

\section{Keywords}

Joubert syndrome; Vermian hypoplasia; Developmental delay; Molar tooth sign

\section{Introduction}

\footnotetext{
oubert syndrome (JS) is considered to be one of the well-known congenital brain malformations which was first described and reported by neurologist Marie Joubert in 1969. She diagnosed five children who had hypoplasia or aplasia of cerebellar vermis and manifested by transient episodic hyperpnea, oculomotor abnormalities, ataxia, and mental retardation ${ }^{[1]}$. Other reported abnormalities include dysmorphic facies, polydactyly, multicystic kidney disease and tongue protrusion. Congenital heart disease, and supratentorial anomaly in the corpus callosum are rare finding $\mathrm{s}^{[2]}$. A characteristic molar tooth appearance is seen with JS in cross-sectional imaging obtained at the level of midbrain and hindbrain, as well as fourth ventricle dilatation, superior cerebellar peduncle thickening and elongation ${ }^{[3]}$.
}

\section{Case Report}

A 6 year-old girl was referred to the pediatric neurology department at our institute as a case of global neurodevelopmental delay, failure to thrive, abnormal intermittent jerky horizontal eye movement, blindness and chronic renal failure on treatment. The patient presented with dyspnea, rapid breath and frequent seizure episodes for one week. The prenatal, natal, post-natal and family histories were unremarkable with two other healthy siblings.

On physical examination, the patient was far below the percentile for her age and sex. Neurological examination demonstrated an ataxic movement, hypotonia, and severe muscle wasting and decreased reflexes without obvious skeletal deformities. Other physical examination findings were normal. 
Reviewing laboratory studies showed significant disturbance in the renal functions. Renal biopsy was performed due to unexplained elevation of renal function tests, and the histopathological correlation demonstrated focal segmental glomerulosclerosis. No genetic testing was performed.

A multiplanar and multi sequential magnetic resonance imaging (MRI) of the brain was ordered and revealed a constellation of abnormalities in the central nervous system in the form of hypoplasia and dysplasia of the cerebellum, deep interpeduncular fossa and abnormally thickened elongated superior cerebellar peduncles giving the molar tooth appearance (Fig. 1). More caudal axial images revealed an absence of cerebellar vermis resulting in a midline cleft between the two cerebellar hemispheres (Fig. 2) as well as the "bat-wing" appearance of the fourth ventricle (Fig. 3). The supratentorial structures, including the corpus callosum appeared normal.

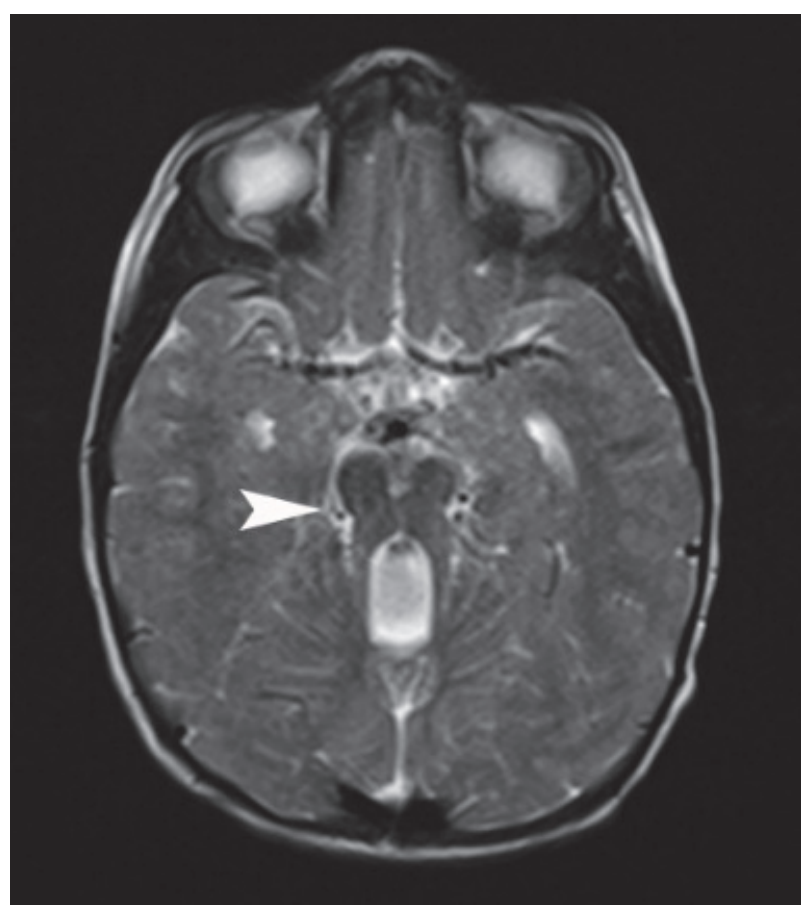

Figure 1. Axial T2-weighted MRI of the brain demonstrates hypoplasia and dysplasia of the cerebellar vermis, thickening and elongation of the superior cerebellar peduncles giving the "molar tooth" appearance (white arrowhead).

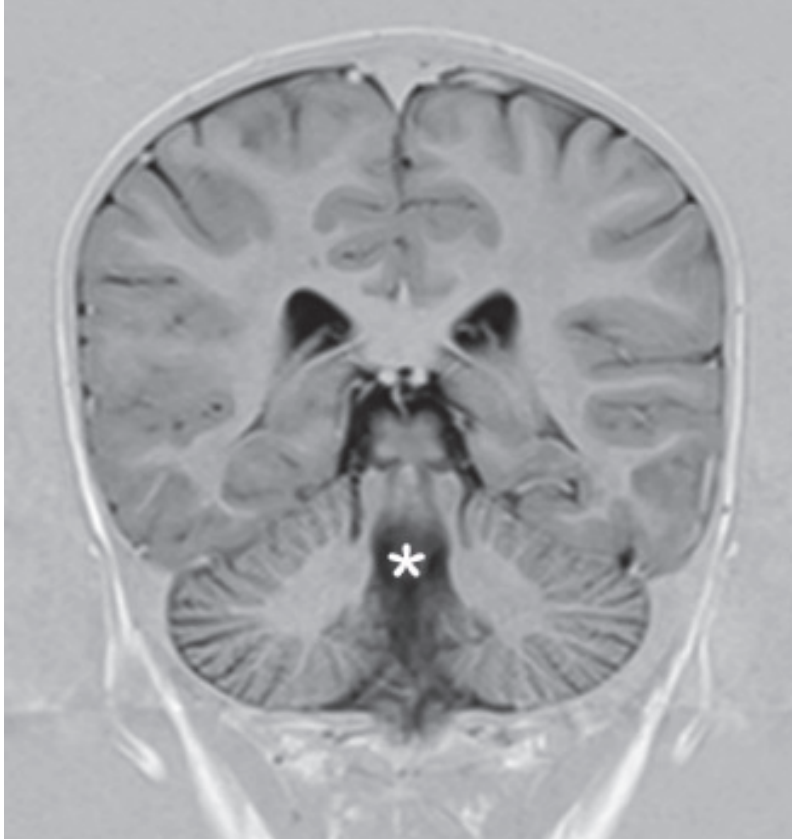

Figure 2. Coronal True inversion recovery T2-weighted MRI of the brain demonstrates a midline defect within the cerebellum (white asterisk) due to absence of cerebellar vermis and enlarged fourth ventricle.

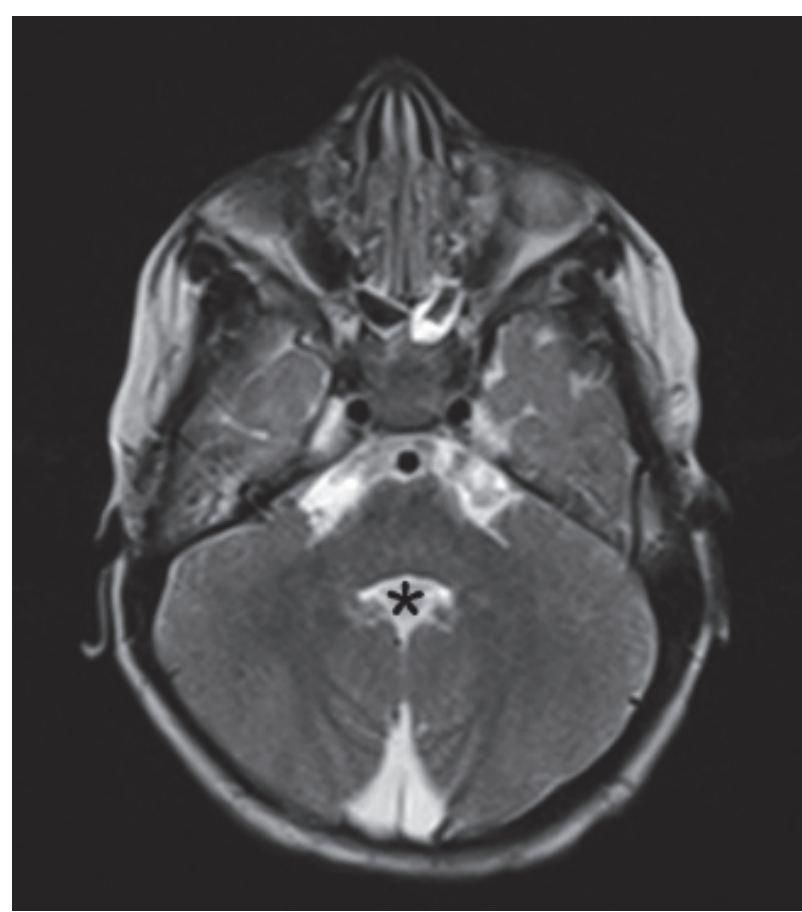

Figure 3. Axial T2-weighted MRI of the brain at the level of middle cerebellar peduncle demonstrates absence of cerebellar vermis resulting in a midline cleft between the two cerebellar hemispheres; "bat-wing" appearance of the fourth ventricle (black asterisk) is also noted. 


\section{Discussion}

Marie Joubert was a neurologist who reported a series of five cases of children with mental retardation, abnormal, deep and rapid breathing, abnormal eye movements, and ataxia, which were associated with agenesis of the cerebellar vermis. The degree of vermian hypoplasia varies across patients ranging from mild to severe ${ }^{[4,5]}$. Cerebellar vermian congenital anomalies have been reported with other disorders besides JS, such as Dandy-Walker syndrome and rhombencephalosynapsis ${ }^{[1,2]}$. The clinical presentation of children with JS may include non-specific features such as hypotonia, ataxia and developmental delay.

Joubert syndrome patients have a variable facial appearance on clinical examination. These features include a large head, a prominent forehead, high rounded eyebrows, ptosis, an upturned nose with evident nostrils, an open mouth, tongue protrusion and rhythmic tongue motions, and occasionally lowset and tilted ears. A postural muscle hypotonia is considered to be one of the hallmark features of $J S^{[6]}$. Severe hypotonia and a lack of spontaneous movement were present in our patient. Respiratory abnormalities have also been considered a 'classic hallmark' of the condition, present in all four siblings initially described by Akcakus and colleagues ${ }^{[6]}$. However, it is not a consistent feature: it was reported in $44 \%$ of the cases by Kendall and colleagues ${ }^{[7]} ; 68 \%$ by Pellegrino et al. ${ }^{[8]}$; $71 \%$ by Maria and colleagues ${ }^{[9]}$. Abnormal respirations are typically seen at the neonatal period but improve with age $\mathrm{e}^{[10,11]}$. Persistent abnormal respiratory symptoms were present in our patient.

Other common pathologic findings include abnormalities of the dentate nuclei, dysplasia of the inferior olivary and paraolivary nuclei, and anomalies of the dorsal column nuclei. An inability of the posterior fossa fiber tracts to cross the midline may be considered as a typical abnormality of this syndrome, although the underlying cause remains unknown ${ }^{[3]}$. Associated supratentorial anomalies are uncommon in JS, but migrational anomaly like cerebral cortical dysplasia and gray matter heterotopias have been reported. Also, some cases revealed parenchymal atrophic changes associated with a mild degree of ventricular dilatation in 6-20\% of cases $^{[1]}$.

Moreover, JS has a very high association with ocular and oculomotor abnormalities, affecting between 70 to $100 \%$ of patients diagnosed with this syndrome, which thus help to make the diagnosis ${ }^{[8,12,13]}$. Our patient had an abnormal eye movement.
Some authors of case reviews of patients with JS have listed the prevalence of additional findings including polydactyly (8\%), ocular coloboma (4\%), and hamartomas of the tongue $(2 \%)^{[14,15]}$. Neuropathological studies reveal aplasia of the cerebellar vermis, malformations of several brainstem nuclei and severe ponto-mesencephalic junction. Extensive posterior fossa involvement with brain stem malformation are considered to be valuable imaging findings that support the diagnosis of JS in combination with the clinical abnormalities in the respiratory system ${ }^{[16]}$.

The term JS and related disorders (JSRD) is used to describe individuals with JS who have additional multi organ involvement including retinal dystrophy, renal disease, ocular colobomas, occipital encephalocele, hepatic fibrosis, polydactyly, oral hamartomas, and endocrine abnormalities ${ }^{[17]}$.

Ocular involvement in patients with JS plays another important role in determining the survival outcome. Those patients who presented without retinal dystrophy have greater survival rates as compared to patients with retinal dystrophy, who have an increased risk of developing multicystic renal disease ${ }^{[1]]}$. Consultation with a nephrologist is usually indicated especially in those patients with renal involvement. Nephronophthisis and severe renal involvement usually require dialysis and organ transplantation early in life. Other complications of end-stage renal disease such as hypertension and anemia require specific treatment and close monitoring. Our patient turned out to have chronic renal disease requiring regular hemodialysis.

Once the diagnosis of JS is suspected, children should be involved in a diagnostic protocol to exclude the presence of other multiorgan involvement. Ocular examinations and referral to the ophthalmology clinic will include different eye tests. These include evaluation of visual acuity, ocular motility, fundus and retinal examination. Hepatorenal imaging and laboratory work up are mandatory. Sonographic assessment of the abdomen will explore the kidneys for small cysts and loss of corticomedullarly differentiation, and the liver for structural abnormalities. Suspicion of hepatic involvement requires further liver imaging techniques, including enhanced MRI for biliary ducts and hepatic parenchyma evaluation. Final diagnosis will be established by liver biopsy.

The developmental outcome in JS varies depending on the involvement of the central nervous system. Steinlin et al. ${ }^{[13]}$ suggested that outcomes in JS can be categorized into three types: children who 
die young; patients who survive, but are severely developmentally delayed and have a variety of visual and motor handicaps; patients whose developmental quotients fall within the mildly delayed range ${ }^{[13]}$. Therefore, in view of the variability of the clinical outcome, it is important to identify the syndrome in the neonatal period so that specified supportive measures can be promptly instituted.

\section{Conclusion}

Joubert syndrome is a rare congenital brain malformation characterized by pathognomonic imaging and clinical findings. Careful imaging evaluation of the brain parenchyma, especially the infra tentorial structures, reveals the unique molar tooth sign and bat-wing appearance of the fourth ventricle, which are essential to establish the diagnosis. JS has variable clinical presentations, multiple associated syndromes, multi-organ involvement and genetic integration. This emphasizes the importance of those radiological scans to reach a timely diagnosis in order to involve multidisciplinary teams early in the treatment of these patients to improve the prognosis. Finally, and most importantly, starting an early genetic counseling is crucial to avoid the risk of recurrence.

\section{Conflict of Interest}

The author has no conflict of interest.

\section{Disclosure}

The author did not received any type of commercial support either in forms of compensation or financial for this study. The author have no financial interest in any of the products or devices, or drugs mentioned in this article.

\section{Ethical Approval}

Obtained.

\section{References}

[1] Osborn AG, Salzman KL, Katzman G, Provenzale J, Castillo M, Hedlund G, Illner A, Harnsberger HR, Cooper J, Jones B, Hamilton B. Diagnostic Imaging: Brain. $1^{\text {st }}$ ed. Salt Lake City: Amirsys, 2004.

[2] van Beek EJ, Majoie CB. Case 25: Joubert syndrome. Radiology 2000; 216(2): 379-382.

[3] McGraw P. The molar tooth sign. Radiology 2003; 229(3): 671-672.
[4] Alorainy IA, Sabir S, Seidahmed MZ, Farooqu HA, Salih MA. Brain stem and cerebellar findings in Joubert syndrome. J Comput Assist Tomogr 2006; 30(1): 116-121.

[5] Poretti A, Huisman TA, Scheer I, Boltshauser E. Joubert syndrome and related disorders: spectrum of neuroimaging findings in 75 patients. AJNR Am J Neuroradiol 2011; 32(8): 1459-1463.

[6] Akcakus M, Gunes T, Kumandas S, Kurtoglu S, Coskun A. Joubert syndrome: Report of a neonatal case. Paediatr Child Health 2003; 8(8): 499-502.

[7] Kendall B, Kingsley D, Lambert SR, Taylor D, Finn P. Joubert syndrome: a clinico-radiological study. Neuroradiology 1990; 31(6): 502-506.

[8] Pellegrino JE, Lensch MW, Muenke M, Chance PF. Clinical and molecular analysis in Joubert syndrome. Am J Med Genet 1997; 72(1): 59-62.

[9] Maria BL, Quisling RG, Rosainz LC, Yachnis AT, Gitten J, Dede $D$, Fennell E. Molar tooth sign in Joubert syndrome: clinical, radiologic, and pathologic significance. J Child Neurol 1999; 14(6): 368-376.

[10] Boltshauser E, Isler W. Joubert syndrome: episodic hyperpnea, abnormal eye movements, retardation and ataxia, associated with dysplasia of the cerebellar vermis. Neuropadiatrie 1977; 8(1): 57-66.

[1 1] Saraiva JM, Baraitser M. Joubert syndrome: a review. Am J Med Genet 1992; 43(4): 726-731.

[12] Maria BL, Hoang KB, Tusa RJ, Mancuso AA, Hamed LM, Quisling RG, Hove MT, Fennell EB, Booth-Jones M, Ringdahl DM, Yachnis AT, Creel G, Frerking B. "Joubert syndrome" revisited: key ocular motor signs with magnetic resonance imaging correlation. J Child Neurol 1997; 12(7): 423-430.

[13] Steinlin M, Schmid M, Landau K, Boltshauser E. Follow-up in children with Joubert syndrome. Neuropediatrics 1997; 28(4): 204-211.

[14] Chance PF, Cavalier L, Satran D, Pellegrino JE, Koenig M, Dobyns WB. Clinical nosologic and genetic aspects of Joubert and related syndromes. J Child Neurol 1999; 14(10): 660-666

[15] Aslan H, Ulker V, Gulcan EM, Numanoglu C, Gul A, Agar M, Ark HC. Prenatal diagnosis of Joubert syndrome: a case report. Prenat Diagn 2002; 22(1): 13-16.

[16] Yachnis AT, Rorke LB. Neuropathology of Joubert syndrome. J Child Neurol 1999; 14(10): 655-659.

[17] Parisi M, Glass I. Joubert Syndrome and Related Disorders. In: GeneReviews ${ }^{\oplus}$. Pagon RA, Adam MP, Ardinger HH, Bird TD, Dolan CR, Fong CT, et al., editors. Seattle (WA): University of Washington, Seattle. 1993-2015. 


\section{الخصائص التصويرية الفريدة للاماغ بمريض يعاني من متلازمة جوبيرت والتصلب الكلوي الكبيبي الجزئي.}

المستخلص. تعتبر متلازمة جوبيرت من أحد الأمر اض الور اثية النادرة التي نورث عن طريق الجين الصبغي الجسدي الكتنحي. تتسم

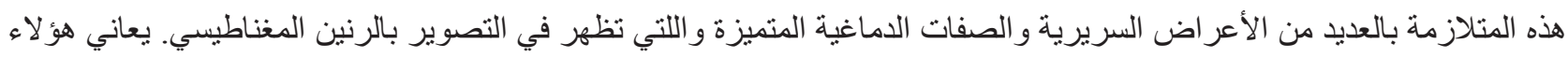

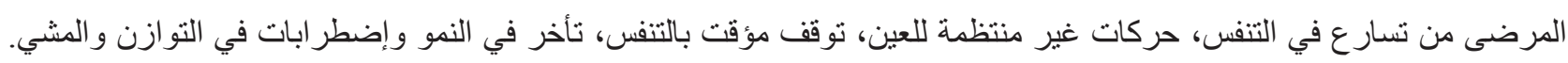

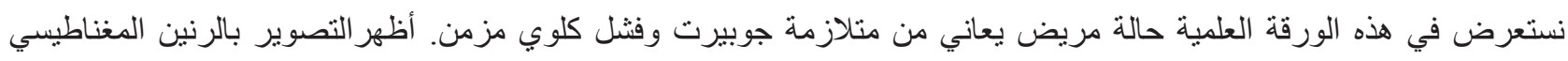

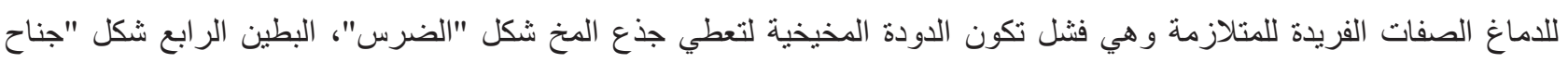

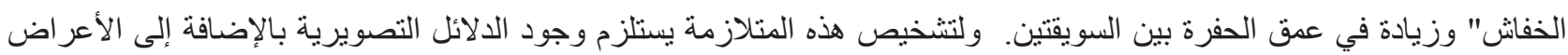
السريرية. 